United Nations Educational, Scientific and Cultural Organization and International Atomic Energy Agency

THE ABDUS SALAM INTERNATIONAL CENTRE FOR THEORETICAL PHYSICS

\title{
INTRODUCTIVE BACKGROUNDS OF MODERN QUANTUM MATHEMATICS WITH APPLICATION TO NONLINEAR DYNAMICAL SYSTEMS
}

\begin{abstract}
The authors dedicate this article to their friend and teacher academician Prof. Anatoliy M. Samoilenko on the occasion of his 70th Birthday with great compliments and gratitude to his brilliant talent and impressive impact to modern theory of nonlinear dynamical systems of mathematical physics and nonlinear analysis.
\end{abstract}

Anatoliy K. Prykarpatsky 1

The AGH University of Science and Technology, Kraków 30-059, Poland, National Academy of Sciences of Ukraine, Lviv, Ukraine and

The Abdus Salam International Centre for Theoretical Physics, Trieste, Italy,

Nikolai N. Bogoliubov Jr2

V.A. Steklov Mathematical Institute of RAN, Moscow, Russian Federation and

The Abdus Salam International Centre for Theoretical Physics, Trieste, Italy,

Jolanta Golenia $3^{3}$

Department of Applied Mathematics, AGH University of Science and Technology, Kraków 30-059, Poland

and

Ufuk Taner眯

Department of Applied Mathematics and Computer Science, Eastern Mediterranean University EMU, Famagusta, North Cyprus and

Kyrenia American University GAU, Institute of Graduate Studies, Kyrenia, North Cyprus.

MIRAMARE - TRIESTE

September 2007

\footnotetext{
${ }^{1}$ pryk.anat@ua.fm, prykanat@cybergal.com

2 nikolai_bogolubov@hotmail.com

$3^{3}$ goljols@tlen.pl

${ }^{4}$ ufuk.taneri@gmail.com
} 


\begin{abstract}
Introductive backgrounds of a new mathematical physics discipline - Quantum Mathematics - are discussed and analyzed both from historical and analytical points of view. The magic properties of the second quantization method, invented by V. Fock in 1934, are demonstrated, and an impressive application to the nonlinear dynamical systems theory is considered.
\end{abstract}




\section{INTRODUCTION}

There is a broad and inclusive view of modern mathematical physics by many mathematicians and mathematical physicists. During the last century, modern mathematical physics evolved within at least four components which illustrate [19] the development of the mathematics and quantum physics synergy:

1) the use of ideas from mathematics in shedding new light on the existing principles of quantum physics, either from a conceptual or from a quantitive point of view;

2 ) the use of ideas from mathematics in discovering new "laws of quantum physics";

3) the use of ideas from quantum physics in shedding new light on existing mathematical structures;

4) the use of ideas from quantum physics in discovering new domains in mathematics.

Each one of these topics plays some role in understanding the modern mathematical physics. However, our success in directions 2) and 4) is certainly more modest than our success in directions 1) and 3). In some cases it is difficult to draw a clear-cut distinction between these two sets. In fact, we are lucky when it is possible to make progress in directions 2) and 4); so much so that when we achieve a major progress, historians like to speak of a revolution. In any case, many of mathematical physicists strive to understand within their research efforts these deep and lofty goals. There are many situations howevere, when mathematical physicists' research efforts are directed toward one other more mundane aspect:

$5)$ the use of ideas from quantum physics and mathematics to benefit "economic competitiveness".

Here too, one might subdivide this aspect into conceptual understanding on one hand (such as the mathematical model of Black and Sholes for pricing of derivative securities in financial markets) and invention on the other: the formulation of new algorithms or materials (e.g. quantum computers) which might revolutionize technology or change our way of life. As in the first four cases, the boundary between these domains is not sharp, and it remains open to views and interpretations. This fifth string can be characterized as "applied" mathematical physics. We will restrict our analysis to the first four strands characterizing modern quantum physics and mathematics aspects; it is believed that that most of the profound applied directions arise after earlier fundamental quantum physics and mathematics progress.

We have passed through an extraordinary 35-year period of development of modern fundamental mathematics and quantum physics. Much of this development has drawn from one subject to understand the other. Not only concepts from diverse fields have been united: statistical physics, quantum field theory and functional integration; gauge theory and geometry; index theory and knot invariants, etc., but also, new phenomena have been recognized and new areas have emerged whose significance for both mathematics and modern quantum physics is only partially understood: for example, non-commutative geometry, superanalysis, mirror symmetry, new topological invariants of manifolds, and the general notion of geometric quantization.

There is no doubt that, over the past 35 years, the ideas from quantum physics have led to far greater inventions of new mathematics than the ideas from mathematics have in discoveries of laws of quantum physics. Recognition of this underlines the opportunities for future progress in the opposite direction: a new understanding of the quantum nature of the world is certainly our expectation! 
Great publicity and recognition has been attached to the progress made in modern geometry, representation theory, and deformation theory due to this interaction. But one should ignore the substantial progress in analysis and probability theory, which unfortunately is more difficult to understand because of its delicate dependence on subtle notions of continuity.

On the other hand, there are deep differences between pure mathematics and modern quantum physics fundamentals. They have evolved from different cultures and they each have a distinctive set of values of their own, suited for their different realms of universality. But both subjects are strongly based on intuition, some natural and some acquired, which form our understanding. Quantum physics describes the natural micro-world. Hence, physicists appeal to observation in order to verify the validity of a physical theory. And, although much of mathematics arises from the natural world, mathematics has no analogous testing grounds - mathematicians appeal to their own set of values, namely mathematical proof, to justify validity of a mathematical theory. In mathematical physics, when announcing results of a mathematical nature, it is necessary to claim a theorem when the proof meets the mathematical community standards for a proof; otherwise, it is necessary to make a conjecture with a detailed outline for support. Most of physics, on the other hand, has completely different standards.

There is no question that the interaction between modern mathematics and quantum physics will change radically during this running century. We do hope however, that this evolution will preserve the positive experience of being a mathematician, a pure physicist, or a mathematical physicist, so that it remains attractive to the brightest and gifted young students today and tomorrow.

It is instructive to look at the beginning of the $X X^{\text {th }}$ century and trace the way mathematics has been exerting influence on modern and classical quantum physics, and next observe the way the modern quantum physics is nowadays exerting so impressive influence on modern mathematics. With the latter, application of modern quantum mathematics to studying nonlinear dynamical systems in functional spaces will for example be a significant topic of our present work. We will begin with a brief history of quantum mathematics:

The beginning of the $X X{ }^{\text {th }}$ century:

- P.A.M. Dirac - first realized and used the fact that the commutator operation $D_{a}: \mathcal{A} \ni b \longrightarrow$ $[a, b] \in \mathcal{A}$, where $a \in \mathcal{A}$ is fixed and $b \in \mathcal{A}$, is a differentiation of any operator algebra $\mathcal{A}$; moreover, he first constructed a spinor matrix realization of the Poincaré symmetry group $\mathcal{P}(1,3)$, 8] $(1920$ 1926);

- J. von Neumann - first applied the spectral theory of self-adjoint operators in Hilbert spaces to explain the radiation spectra of atoms and the stability of the related matter, [30] (1926);

- V. Fock - first introduced the notion of many-particle Hilbert space, Fock space, and introduced the related creation and annihilation operators acting in it, 13] (1932);

- H. Weyl - first understood the fundamental role of the notion of symmetry in physics and developed a physics-oriented group theory; moreover he showed the importance of different representations of classical matrix groups for physics and studied the unitary representations of the Heisenberg-Weyl group related with creation and annihilation operators in Fock space, 33] (1931). 
The end of the $X X^{\text {th }}$ century:

New developments are due to

- L. Faddeev with co-workers - quantum inverse spectral theory transform, [10] (1978);

- V. Drinfeld, S. Donaldson, E. Witten - quantum groups and algebras, quantum topology, quantum super-analysis, [21, 22, 34] (1982-1994);

- Yu. Manin, R. Feynman - quantum information theory, [28, 11, 12] (1980-1986);

- P. Shor, E. Deutsch, L. Grover and others - quantum computer algorithms, 32, 20, 18, (19851997).

As one can observe, many exciting and highly important mathematical achievements were strictly motivated by the impressive and deep influence of quantum physics ideas and ways of thinking, leading nowadays to an altogether new scientific field often called quantum mathematics.

Following this quantum mathematical way of thinking, we will demonstrate below that a wide class of strictly nonlinear dynamical systems in functional spaces can be treated as a natural object in specially constructed Fock spaces in which the corresponding evolution flows are completely linearized. Thereby, the powerful machinery of classical mathematical tools can be applied to studying the analytical properties of exact solutions to suitably well posed Cauchy problems.

\section{Mathematical preliminaries: Fock Space And its Realizations}

Let $\Phi$ be a separable Hilbert space, $F$ be a topological real linear space and $\mathcal{A}:=\{A(\varphi): \varphi \in F\}$ a family of commuting self-adjoint operators in $\Phi$ (i.e. these operators commute in the sense of their resolutions of the identity). Consider the Gelfand rigging [2] of the Hilbert space $\Phi$, i.e., a chain

$$
\mathcal{D} \subset \Phi_{+} \subset \Phi \subset \Phi_{-} \subset \mathcal{D}^{\prime}
$$

in which $\Phi_{+}$and $\Phi_{-}$are further Hilbert spaces, and the inclusions are dense and continuous, i.e. $\Phi_{+}$is topologically (densely and continuously) and quasi-nuclearly (the inclusion operator $i: \Phi_{+} \longrightarrow \Phi$ is of the Hilbert - Schmidt type) embedded into $\Phi, \Phi_{-}$is the dual of $\Phi_{+}$with respect to the scalar product $<., .>_{\Phi}$ in $\Phi$, and $\mathcal{D}$ is a separable projective limit of Hilbert spaces, topologically embedded into $\Phi_{+}$. Then, the following structural theorem [2, 3] holds:

Theorem 2.1. Assume that the family of operators $\mathcal{A}$ satisfies the following conditions:

a) $\mathcal{D} \subset \operatorname{Dom} A(\varphi), \varphi \in F$, and the closure of the operator $A(\varphi) \uparrow \mathcal{D}$ coincides with $A(\varphi)$ for any $\varphi \in F$, that is $A(\varphi) \uparrow \mathcal{D}=A(\varphi)$ in $\Phi$;

b) the Range $A(\varphi) \uparrow \mathcal{D} \subset \Phi_{+}$for any $\varphi \in F$;

c) for every $f \in \mathcal{D}$ the mapping $F \ni \varphi \longrightarrow A(\varphi) f \in \Phi_{+}$is linear and continuous;

d) there exists a strong cyclic (vacuum) vector $|\Omega\rangle \in \bigcap_{\varphi \in F} \operatorname{Dom} A(\varphi)$, such that the set of all vectors $|\Omega\rangle, \prod_{j=1}^{n} A\left(\varphi_{j}\right)|\Omega\rangle, n \in \mathbb{Z}_{+}$, is total in $\Phi_{+}$(i.e. their linear hull is dense in $\left.\Phi_{+}\right)$.

Then there exists a probability measure $\mu$ on $\left(F^{\prime}, C_{\sigma}\left(F^{\prime}\right)\right)$, where $F^{\prime}$ is the dual of $F$ and $C_{\sigma}\left(F^{\prime}\right)$ is the $\sigma$-algebra generated by cylinder sets in $F^{\prime}$ such that, for $\mu$-almost every $\eta \in F^{\prime}$ there is a generalized 
joint eigenvector $\omega(\eta) \in \Phi_{-}$of the family $\mathcal{A}$, corresponding to the joint eigenvalue $\eta \in F^{\prime}$, that is

$$
<\omega(\eta), A(\varphi) f>_{\Phi}=\eta(\varphi)<\omega(\eta), f>_{\Phi}
$$

with $\eta(\varphi) \in \mathbb{R}$ denoting the pairing between $F$ and $F^{\prime}$.

The mapping

$$
\Phi_{+} \ni f \longrightarrow<\omega(\eta), f>_{\Phi}:=\hat{f}(\eta) \in \mathbb{C}
$$

for any $\eta \in F^{\prime}$ can be continuously extended to a unitary surjective operator $\mathcal{F}: \Phi \longrightarrow L_{2}^{(\mu)}\left(F^{\prime} ; \mathbb{C}\right)$, where

$$
\mathcal{F} f(\eta):=\hat{f}(\eta)
$$

for any $\eta \in F^{\prime}$ is a generalized Fourier transform, corresponding to the family $\mathcal{A}$. Moreover, the image of the operator $A(\varphi), \varphi \in F^{\prime}$, under the $\mathcal{F}$-mapping is the operator of multiplication by the function $F^{\prime} \ni \eta \rightarrow \eta(\varphi) \in \mathbb{C}$.

We assume additionally that the main Hilbert space $\Phi$ possesses the standard Fock space (bose)structure [4, 6, 31, that is

$$
\Phi=\oplus_{n \in \mathbb{Z}_{+}} \Phi_{n}
$$

where subspaces $\Phi_{n}:=\Phi_{(s)}^{\otimes n}, n \in \mathbb{Z}_{+}$, are the symmetrized tensor products of a Hilbert space $\mathcal{H}:=$ $L_{2}\left(\mathbb{R}^{m} ; \mathbb{C}\right)$. If a vector $g:=\left(g_{0}, g_{1}, \ldots, g_{n}, \ldots\right) \in \Phi$, its norm

$$
\|g\|_{\Phi}:=\left(\sum_{n \in \mathbb{Z}_{+}}\left\|g_{n}\right\|_{n}^{2}\right)^{1 / 2}
$$

where $g_{n} \in \Phi_{(s)}^{\otimes n} \simeq L_{2,(s)}\left(\left(\mathbb{R}^{m}\right)^{n} ; \mathbb{C}\right)$ and $\|\ldots\|_{n}$ is the corresponding norm in $\Phi_{(s)}^{\otimes n}$ for all $n \in \mathbb{Z}_{+}$. Denote here that, concerning the rigging structure (2.1), there holds the corresponding rigging for the Hilbert spaces $\Phi_{(s)}^{\otimes n}, n \in \mathbb{Z}_{+}$, that is

$$
\mathcal{D}_{(s)}^{n} \subset \Phi_{(s),+}^{\otimes n} \subset \Phi_{(s)}^{\otimes n} \subset \Phi_{(s),-}^{\otimes n}
$$

with some suitably chosen dense and separable topological spaces of symmetric functions $\mathcal{D}_{(s)}^{n}, n \in \mathbb{Z}_{+}$. Concerning expansion (2.5) we obtain by means of projective and inductive limits [2, 6, 3] the quasi-nucleous rigging of the Fock space $\Phi$ in the form (2.1):

$$
\mathcal{D} \subset \Phi_{+} \subset \Phi \subset \Phi_{-} \subset \mathcal{D}^{\prime}
$$

Consider now any vector $\left|(\alpha)_{n}\right\rangle \in \Phi_{(s)}^{\otimes n}, n \in \mathbb{Z}_{+}$, which can be written [2, 4, 26] in the following canonical Dirac ket-form:

$$
\left|(\alpha)_{n}\right\rangle:=\left|\alpha_{1}, \alpha_{2}, \ldots, \alpha_{n}\right\rangle
$$

where, by definition,

$$
\left|\alpha_{1}, \alpha_{2}, \ldots, \alpha_{n}\right\rangle:=\frac{1}{\sqrt{n !}} \sum_{\sigma \in S_{n}}\left|\alpha_{\sigma(1)}\right\rangle \otimes\left|\alpha_{\sigma(2)}\right\rangle \ldots\left|\alpha_{\sigma(n)}\right\rangle
$$


and $\left|\alpha_{j}\right\rangle \in \Phi_{(s)}^{\otimes 1}\left(\mathbb{R}^{m} ; \mathbb{C}\right):=\mathcal{H}$ for any fixed $j \in \mathbb{Z}_{+}$. The corresponding scalar product of base vectors as (2.9) is given as follows:

$$
\begin{gathered}
\left\langle(\beta)_{n} \mid(\alpha)_{n}\right\rangle:=\left\langle\beta_{n}, \beta_{n-1}, \ldots, \beta_{2}, \beta_{1} \mid \alpha_{1}, \alpha_{2}, \ldots, \alpha_{n-1}, \alpha_{n}\right\rangle \\
=\sum_{\sigma \in S_{n}}\left\langle\beta_{1} \mid \alpha_{\sigma(1)}\right\rangle \ldots\left\langle\beta_{n} \mid \alpha_{\sigma(n)}\right\rangle:=\operatorname{per}\left\{\left\langle\beta_{i} \mid \alpha_{j}\right\rangle: i, j=\overline{1, n}\right\},
\end{gathered}
$$

where "per" denotes the permanent of matrix and $\langle. \mid$.$\rangle is the corresponding product in the Hilbert space$ $\mathcal{H}$. Based now on representation (2.8) one can define an operator $a^{+}(\alpha): \Phi_{(s)}^{\otimes n} \longrightarrow \Phi_{(s)}^{\otimes(n+1)}$ for any $|\alpha\rangle \in \mathcal{H}$ as follows:

$$
a^{+}(\alpha)\left|\alpha_{1}, \alpha_{2}, \ldots, \alpha_{n}\right\rangle:=\left|\alpha, \alpha_{1}, \alpha_{2}, \ldots, \alpha_{n}\right\rangle,
$$

which is called the "creation" operator in the Fock space $\Phi$. The adjoint operator $a(\beta):=\left(a^{+}(\beta)\right)^{*}$ : $\Phi_{(s)}^{\otimes(n+1)} \longrightarrow \Phi_{(s)}^{\otimes n}$ with respect to the Fock space $\Phi(2.5)$ for any $|\beta\rangle \in \mathcal{H}$, called the "annihilation" operator, acts as follows:

$$
a(\beta)\left|\alpha_{1}, \alpha_{2}, \ldots, \alpha_{n+1}\right\rangle:=\sum_{\sigma \in S_{n}}\left\langle\beta, \alpha_{j}\right\rangle\left|\alpha_{1}, \alpha_{2}, \ldots, \alpha_{j-1}, \hat{\alpha}_{j}, \alpha_{j+1}, \ldots, \alpha_{n+1}\right\rangle,
$$

where the "hat" over a vector denotes that it should be omitted from the sequence.

It is easy to check that the commutator relationship

$$
\left[a^{+}(\alpha), a(\beta)\right]=\langle\alpha, \beta\rangle
$$

holds for any vectors $|\alpha\rangle \in \mathcal{H}$ and $|\beta\rangle \in \mathcal{H}$. Expression (2.13), owing to the rigged structure (2.1), can be naturally extended to the general case, when vectors $|\alpha\rangle$ and $|\beta\rangle \in \mathcal{H}_{-}$, conserving its form. In particular, taking $|\alpha\rangle:=|\alpha(x)\rangle=\frac{1}{\sqrt{2 \pi}} e^{i\langle\lambda, x\rangle} \in \mathcal{H}_{-}:=L_{2,-}\left(\mathbb{R}^{m} ; \mathbb{C}\right)$ for any $x \in \mathbb{R}^{m}$, one easily gets from (2.13) that

$$
\left[a^{+}(x), a(y)\right]=\delta(x-y)
$$

where we put, by definition, $a^{+}(x):=a^{+}(\alpha(x))$ and $a(y):=a(\alpha(y))$ for all $x, y \in \mathbb{R}^{m}$ and denoted by $\delta(\cdot)$ the classical Dirac delta-function.

The construction above makes it possible to observe easily that there exists a unique vacuum vector $|\Omega\rangle \in \mathcal{H}_{+}$, such that for any $x \in \mathbb{R}^{m}$

$$
a(x)|\Omega\rangle=0,
$$

and the set of vectors

$$
\left(\prod_{j=1}^{n} a^{+}\left(x_{j}\right)\right)|\Omega\rangle \in \Phi_{(s)}^{\otimes n}
$$

is total in $\Phi_{(s)}^{\otimes n}$, that is their linear integral hull over the dual functional spaces $\hat{\Phi}_{(s)}^{\otimes n}$ is dense in the Hilbert space $\Phi_{(s)}^{\otimes n}$ for every $n \in \mathbb{Z}_{+}$. This means that for any vector $g \in \Phi$ the following representation

$$
g=\oplus_{n \in \mathbb{Z}_{+}} \int_{\left(\mathbb{R}^{m}\right)^{n}} \hat{g}_{n}\left(x_{1}, \ldots, x_{n}\right) a^{+}\left(x_{1}\right) a^{+}\left(x_{2}\right) \ldots a^{+}\left(x_{n}\right)|\Omega\rangle
$$

holds with the Fourier type coefficients $\hat{g}_{n} \in \hat{\Phi}_{n}:=\hat{\Phi}_{(s)}^{\otimes n}$ for all $n \in \mathbb{Z}_{+}$, with $\hat{\Phi}_{(s)}^{\otimes 1}:=\mathcal{H} \simeq L_{2}\left(\mathbb{R}^{m} ; \mathbb{C}\right)$. The latter is naturally endowed with the dual to (2.1) Gelfand type quasi-nucleous rigging

$$
\mathcal{H}_{+} \subset \mathcal{H} \subset \mathcal{H}
$$


making it possible to construct a quasi-nucleous rigging of the dual Fock space $\hat{\Phi}:=\oplus_{n \in \mathbb{Z}_{+}} \hat{\Phi}_{n}$. Thereby, chain (2.18) generates the dual Fock space quasi-nucleous rigging

$$
\hat{\mathcal{D}} \subset \hat{\Phi}_{+} \subset \hat{\Phi} \subset \hat{\Phi}_{-} \subset \hat{\mathcal{D}}^{\prime}
$$

with respect to the central Fock type Hilbert space $\hat{\Phi}$, where $\hat{\mathcal{D}} \simeq \mathcal{D}$, easily following from (2.1) and (2.18).

Construct now the following self-adjoint operator

$$
a^{+}(x) a(x):=\rho(x): \Phi \rightarrow \Phi,
$$

called the density operator at a point $x \in \mathbb{R}^{m}$, satisfying the commutation properties:

$$
\begin{gathered}
{[\rho(x), \rho(y)]=0,} \\
{[\rho(x), a(y)]=-a(y) \delta(x-y),} \\
{\left[\rho(x), a^{+}(y)\right]=a^{+}(y) \delta(x-y)}
\end{gathered}
$$

for all $y \in \mathbb{R}^{m}$.

Now, if to construct the following self-adjoint family $\mathcal{A}:=\left\{\int_{\mathbb{R}^{m}} \rho(x) \varphi(x) d x: \varphi \in F\right\}$ of linear operators in the Fock space $\Phi$, where $F:=\mathcal{S}\left(\mathbb{R}^{m} ; \mathbb{R}\right)$ is the Schwartz functional space, one can derive, making use of Theorem 2.1, that there exists the generalized Fourier transform (2.4), such that

$$
\Phi(\mathcal{H})=L_{2}^{(\mu)}\left(\mathcal{S}^{\prime} ; \mathbb{C}\right) \simeq \int_{\mathcal{S}^{\prime}}^{\oplus} \Phi_{\eta} d \mu(\eta)
$$

for some Hilbert space sets $\Phi_{\eta}, \eta \in F^{\prime}$, and a suitable measure $\mu$ on $\mathcal{S}^{\prime}$, with respect to which the corresponding joint eigenvector $\omega(\eta) \in \Phi_{+}$for any $\eta \in F^{\prime}$ generates the Fourier transformed family $\hat{\mathcal{A}}=\{\eta(\varphi) \in \mathbb{R}: \varphi \in \mathcal{S}\}$. Moreover, if $\operatorname{dim} \Phi_{\eta}=1$ for all $\eta \in F$, the Fourier transformed eigenvector $\hat{\omega}(\eta):=\Omega(\eta)=1$ for all $\eta \in F^{\prime}$.

Now we will consider the family of self-adjoint operators $\mathcal{A}$ as generating a unitary family $\mathcal{U}:=$ $\{U(\varphi): \varphi \in F\}=\exp (i \mathcal{A})$, where for any $\rho(\varphi) \in \mathcal{A}, \varphi \in F$, the operator

$$
U(\varphi):=\exp [i \rho(\varphi)]
$$

is unitary, satisfying the abelian commutation condition

$$
U\left(\varphi_{1}\right) U\left(\varphi_{2}\right)=U\left(\varphi_{1}+\varphi_{2}\right)
$$

for any $\varphi_{1}, \varphi_{2} \in F$.

Since, in general, the unitary family $\mathcal{U}=\exp (i \mathcal{A})$ is defined in some Hilbert space $\Phi$, not necessarily being of Fock type, the important problem of describing its Hilbertian cyclic representation spaces arises, within which the factorization

$$
\rho(\varphi)=\int_{\mathbb{R}^{m}} a^{+}(x) a(x) \varphi(x) d x
$$

jointly with relationships (2.21) hold for any $\varphi \in F$. This problem can be treated using mathematical tools devised both within the representation theory of $C^{*}$-algebras [9] and the Gelfand-Vilenkin [14] approach. Below we will describe the main features of the Gelfand-Vilenkin formalism, being much more suitable for the task, providing a reasonably unified framework of constructing the corresponding representations. 
Definition 2.2. Let $F$ be a locally convex topological vector space, $F_{0} \subset F$ be a finite dimensional subspace of $F . \quad$ Let $F^{0} \subseteq F^{\prime}$ be defined by

$$
F^{0}:=\left\{\xi \in F^{\prime}:\left.\quad \xi\right|_{F_{0}}=0\right\}
$$

and called the annihilator of $F_{0}$.

The quotient space $F^{\prime 0}:=F^{\prime} / F^{0}$ may be identified with $F_{0}^{\prime} \subset F^{\prime}$, the adjoint space of $F_{0}$.

Definition 2.3. Let $A \subseteq F^{\prime}$; then the subset

$$
X_{F^{0}}^{(A)}:=\left\{\xi \in F^{\prime}: \xi+F^{0} \subset A\right\}
$$

is called the cylinder set with base $A$ and generating subspace $F^{0}$.

Definition 2.4. Let $n=\operatorname{dim} F_{0}=\operatorname{dim} F_{0}^{\prime}=\operatorname{dim} F^{\prime 0}$. One says that a cylinder set $X^{(A)}$ has Borel base, if $A$ is Borel, when regarded as a subset of $\mathbb{R}^{n}$.

The family of cylinder sets with Borel base forms an algebra of sets.

Definition 2.5. The measurable sets in $F^{\prime}$ are the elements of the $\sigma$ - algebra generated by the cylinder sets with Borel base.

Definition 2.6. A cylindrical measure in $F^{\prime}$ is a real-valued $\sigma$-pre-additive function $\mu$ defined on the algebra of cylinder sets with Borel base and satisfying the conditions $0 \leq \mu(X) \leq 1$ for any $X, \mu\left(F^{\prime}\right)=1$ and $\mu\left(\coprod_{j \in \mathbb{Z}_{+}} X_{j}\right)=\sum_{j \in \mathbb{Z}_{+}} \mu\left(X_{j}\right)$, if all sets $X_{j} \subset F^{\prime}, j \in \mathbb{Z}_{+}$, have a common generating subspace $F_{0} \subset F$.

Definition 2.7. A cylindrical measure $\mu$ satisfies the commutativity condition if and only if for any bounded continuous function $\alpha: \mathbb{R}^{n} \longrightarrow \mathbb{R}$ of $n \in \mathbb{Z}_{+}$real variables the function

$$
\alpha\left[\varphi_{1}, \varphi_{2}, \ldots, \varphi_{n}\right]:=\int_{F^{\prime}} \alpha\left(\eta\left(\varphi_{1}\right), \eta\left(\varphi_{2}\right), \ldots, \eta\left(\varphi_{n}\right)\right) d \mu(\eta)
$$

is sequentially continuous in $\varphi_{j} \in F, j=\overline{1, m}$. (It is well known [14, 15] that in countably normed spaces the properties of sequential and ordinary continuity are equivalent).

Definition 2.8. A cylindrical measure $\mu$ is countably additive if and only if for any cylinder set $X=$ $\coprod_{j \in \mathbb{Z}_{+}} X_{j}$, which is the union of countably many mutually disjoints cylinder sets $X_{j} \subset F^{\prime}, j \in \mathbb{Z}_{+}$, $\mu(X)=\sum_{j \in \mathbb{Z}_{+}} \mu\left(X_{j}\right)$.

The following propositions hold.

Proposition 2.9. A countably additive cylindrical measure $\mu$ can be extended to a countably additive measure on the $\sigma$ - algebra, generated by the cylinder sets with Borel base. Such a measure will also be called a cylindrical measure.

Proposition 2.10. Let $F$ be a nuclear space. Then any cylindrical measure $\mu$ on $F^{\prime}$, satisfying the continuity condition, is countably additive. 
Definition 2.11. Let $\mu$ be a cylindrical measure in $F^{\prime}$. The Fourier transform of $\mu$ is the nonlinear functional

$$
\mathcal{L}(\varphi):=\int_{F^{\prime}} \exp [i \eta(\varphi)] d \mu(\eta)
$$

Definition 2.12. The nonlinear functional $\mathcal{L}: F \longrightarrow \mathbb{C}$ on $F$, defined by (2.29), is called positive definite, if and only if for all $f_{j} \in F$ and $\lambda_{j} \in \mathbb{C}, j=\overline{1, n}$, the condition

$$
\sum_{j, k=1}^{n} \bar{\lambda}_{j} \mathcal{L}\left(f_{k}-f_{j}\right) \lambda_{k} \geq 0
$$

holds for any $n \in \mathbb{Z}_{+}$.

Proposition 2.13. The functional $\mathcal{L}: F \longrightarrow \mathbb{C}$ on $F$, defined by (2.29), is the Fourier transform of a cylindrical measure on $F^{\prime}$, if and only if it is positive definite, sequentially continuous and satisfying the condition $\mathcal{L}(0)=1$.

Suppose now that we have a continuous unitary representation of the unitary family $\mathcal{U}$ in a Hilbert space $\Phi$ with a cyclic vector $|\Omega\rangle \in \Phi$. Then we can put

$$
\mathcal{L}(\varphi):=\langle\Omega|U(\varphi)| \Omega\rangle
$$

for any $\varphi \in F:=\mathcal{S}$, being the Schwartz space on $\mathbb{R}^{m}$, and observe that functional (2.31) is continuous on $F$ owing to the continuity of the representation. Therefore, this functional is the generalized Fourier transform of a cylindrical measure $\mu$ on $\mathcal{S}^{\prime}$ :

$$
\langle\Omega|U(\varphi)| \Omega\rangle=\int_{\mathcal{S}^{\prime}} \exp [i \eta(\varphi)] d \mu(\eta) .
$$

From the spectral point of view, based on Theorem 2.1 there is an isomorphism between the Hilbert spaces $\Phi$ and $L_{2}^{(\mu)}\left(\mathcal{S}^{\prime} ; \mathbb{C}\right)$, defined by $|\Omega\rangle \longrightarrow \Omega(\eta)=1$ and $U(\varphi)|\Omega\rangle \longrightarrow \exp [i \eta(\varphi)]$ and next extended by linearity upon the whole Hilbert space $\Phi$.

In the case of the non-cyclic case there exists a finite or countably infinite family of measures $\left\{\mu_{k}: k \in \mathbb{Z}_{+}\right\}$ on $\mathcal{S}^{\prime}$, with $\Phi \simeq \oplus_{k \in \mathbb{Z}_{+}} L_{2}^{\left(\mu_{k}\right)}\left(\mathcal{S}^{\prime} ; \mathbb{C}\right)$ and the unitary operator $U(\varphi): \Phi \longrightarrow \Phi$ for any $\varphi \in \mathcal{S}^{\prime}$ corresponds in all $L_{2}^{\left(\mu_{k}\right)}\left(\mathcal{S}^{\prime} ; \mathbb{C}\right), k \in \mathbb{Z}_{+}$, to $\exp [i \eta(\varphi)]$. This means that there exists a single cylindrical measure $\mu$ on $\mathcal{S}^{\prime}$ and a $\mu$-measurable field of Hilbert spaces $\Phi_{\eta}$ on $\mathcal{S}^{\prime}$, such that

$$
\Phi \simeq \int_{\mathcal{S}^{\prime}}^{\oplus} \Phi_{\eta} d \mu(\eta)
$$

with $U(\varphi): \Phi \longrightarrow \Phi$, corresponding [14] to the operator of multiplication by $\exp [i \eta(\varphi)]$ for any $\varphi \in \mathcal{S}$ and $\eta \in \mathcal{S}^{\prime}$. Thereby, having constructed the nonlinear functional (2.29) in an exact analytical form, one can retrieve the representation of the unitary family $\mathcal{U}$ in the corresponding Hilbert space $\Phi$ of the Fock type, making use of the suitable factorization (2.25) as follows: $\Phi=\oplus_{n \in \mathbb{Z}_{+}} \Phi_{n}$, where

$$
\Phi_{n}=\underset{f_{n} \in L_{2, s}\left(\left(R^{m}\right)^{n} ; \mathbb{C}\right)}{\operatorname{span}}\left\{\prod_{j=1, n} a^{+}\left(x_{j}\right)|\Omega\rangle\right\},
$$

for all $n \in \mathbb{Z}_{+}$. The cyclic vector $|\Omega\rangle \in \Phi$ can be, in particular, obtained as the ground state vector of some unbounded self-adjoint positive definite Hamilton operator $\mathbb{H}: \Phi \longrightarrow \Phi$, commuting with the self-adjoint 
particles number operator

$$
\mathbb{N}:=\int_{\mathbb{R}^{m}} \rho(x) d x,
$$

that is $[\mathbb{H}, \mathbb{N}]=0$. Moreover, the conditions

$$
\mathbb{H}|\Omega\rangle=0
$$

and

$$
\inf _{g \in \text { dom } \mathbb{H}}\langle g, \mathbb{H} g\rangle=\langle\Omega|\mathbb{H}| \Omega\rangle=0
$$

hold for the operator $\mathbb{H}: \Phi \longrightarrow \Phi$, where dom $\mathbb{H}$ denotes its domain of definition.

To find the functional (2.31), which is called the generating Bogolubov type functional for moment distribution functions

$$
F_{n}\left(x_{1}, x_{2}, \ldots, x_{n}\right):=\left\langle\Omega\left|: \rho\left(x_{1}\right) \rho\left(x_{2}\right) \ldots \rho\left(x_{n}\right):\right| \Omega\right\rangle,
$$

where $x_{j} \in \mathbb{R}^{m}, j=\overline{1, n}$, and the normal ordering operation $: \cdot:$ is defined as

$$
: \rho\left(x_{1}\right) \rho\left(x_{2}\right) \ldots \rho\left(x_{n}\right):=\prod_{j=1}^{n}\left(\rho\left(x_{j}\right)-\sum_{k=1}^{j} \delta\left(x_{j}-x_{k}\right)\right),
$$

it is convenient to choose the Hamilton operator $\mathbb{H}: \Phi \longrightarrow \Phi$ in the following [16, 15, [5] algebraic form:

$$
\mathbb{H}:=\frac{1}{2} \int_{\mathbb{R}^{m}} K^{+}(x) \rho^{-1}(x) K(x) d x+V(\rho),
$$

being equivalent in the Hilbert space $\Phi$ to the positive definite operator expression

$$
\mathbb{H}:=\frac{1}{2} \int_{\mathbb{R}^{m}}\left(K^{+}(x)-A(x ; \rho)\right) \rho^{-1}(x)(K(x)-A(x ; \rho)) d x,
$$

where $A(x ; \rho): \Phi \rightarrow \Phi, \quad x \in \mathbb{R}^{m}$, is some specially chosen linear self-adjoint operator. The "potential" operator $V(\rho): \Phi \longrightarrow \Phi$ is, in general, a polynomial (or analytical) functional of the density operator $\rho(x): \Phi \longrightarrow \Phi$ and the operator is given as

$$
K(x):=\nabla_{x} \rho(x) / 2+i J(x)
$$

where the self-adjoint "current" operator $J(x): \Phi \longrightarrow \Phi$ can be defined (but non-uniquely) from the equality

$$
\partial \rho / \partial t=\frac{1}{i}[\mathbb{H}, \rho(x)]=-<\nabla_{x} \cdot J(x)>,
$$

holding for all $x \in \mathbb{R}^{m}$. Such an operator $J(x): \Phi \longrightarrow \Phi, x \in \mathbb{R}^{m}$ can exist owing to the commutation condition $[\mathbb{H}, \mathbb{N}]=0$, giving rise to the continuity relationship (2.43), if taking into account that supports supp $\rho$ of the density operator $\rho(x): \Phi \longrightarrow \Phi, x \in \mathbb{R}^{m}$, can be chosen arbitrarily owing to the independence of (2.43) on the potential operator $V(\rho): \Phi \longrightarrow \Phi$, but its strict dependence on the corresponding representation (2.33). Denote also that representation (2.41) holds only under the condition that there exists such a self-adjoint operator $A(x ; \rho): \Phi \longrightarrow \Phi, x \in \mathbb{R}^{m}$, that

$$
K(x)|\Omega\rangle=A(x ; \rho)|\Omega\rangle
$$

for all ground states $|\Omega\rangle \in \Phi$, correspond to suitably chosen potential operators $V(\rho): \Phi \longrightarrow \Phi$. 
The self-adjointness of the operator $A(x ; \rho): \Phi \longrightarrow \Phi, x \in \mathbb{R}^{m}$, can be stated following schemes from works [16, 5], under the additional condition of the existence of such a linear anti-unitary mapping $T: \Phi \longrightarrow \Phi$ that the following invariance conditions hold:

$$
T \rho(x) T^{-1}=\rho(x), \quad T J(x) T^{-1}=-J(x), \quad T|\Omega\rangle=|\Omega\rangle
$$

for any $x \in \mathbb{R}^{m}$. Thereby, owing to conditions (2.45), the following expressions

$$
K^{*}(x)|\Omega\rangle=A(x ; \rho)|\Omega\rangle=K(x)|\Omega\rangle
$$

hold for any $x \in \mathbb{R}^{m}$, giving rise to the self-adjointness of the operator $A(x ; \rho): \Phi \longrightarrow \Phi, x \in \mathbb{R}^{m}$.

Based now on the construction above one easily deduces from expression (2.43) that the generating Bogolubov type functional (2.31) obeys for all $x \in \mathbb{R}^{m}$ the following functional-differential equation:

$$
\left[\nabla_{x}-i \nabla_{x} \varphi\right] \frac{1}{2 i} \frac{\delta \mathcal{L}(\varphi)}{\delta \varphi(x)}=A\left(x ; \frac{1}{i} \frac{\delta}{\delta \varphi}\right) \mathcal{L}(\varphi)
$$

whose solutions should satisfy the Fourier transform representation (2.32). In particular, a wide class of special so-called Poissonian white noise type solutions to the functional-differential equation (2.47) was obtained in [16, 5] by means of functional-operator methods in the following generalized form:

$$
\mathcal{L}(\varphi)=\exp \left\{A\left(\frac{1}{i} \frac{\delta}{\delta \varphi}\right)\right\} \exp \left(\bar{\rho} \int_{\mathbb{R}^{m}}\{\exp [i \varphi(x)]-1\} d x\right)
$$

where $\bar{\rho}:=\langle\Omega|\rho| \Omega\rangle \in \mathbb{R}_{+}$is a Poisson distribution density parameter.

Consider now the case, when the basic Fock space $\Phi=\otimes_{j=1}^{s} \Phi^{(j)}$, where $\Phi^{(j)}, j=\overline{1, s}$, are Fock spaces corresponding to the different types of independent cyclic vectors $\left|\Omega_{j}\right\rangle \in \Phi^{(j)}, j=\overline{1, s}$. This, in particular, means that the suitably constructed creation and annihilation operators $a_{j}(x), a_{k}^{+}(y): \Phi \longrightarrow \Phi, j, k=\overline{1, s}$, satisfy the following commutation relations:

$$
\begin{gathered}
{\left[a_{j}(x), a_{k}(y)\right]=0,} \\
{\left[a_{j}(x), a_{k}^{+}(y)\right]=\delta_{j k} \delta(x-y)}
\end{gathered}
$$

for any $x, y \in \mathbb{R}^{m}$.

Definition 2.14. A vector $|u\rangle \in \Phi, x \in \mathbb{R}^{m}$, is called coherent with respect to a mapping $u \in L_{2}\left(\mathbb{R}^{m} ; \mathbb{R}^{s}\right):=$ $M$, if it satisfies the eigenfunction condition

$$
a_{j}(x)|u\rangle=u_{j}(x)|u\rangle
$$

for each $j=\overline{1, s}$ and all $x \in \mathbb{R}^{m}$.

It is easy to check that the coherent vectors $|u\rangle \in \Phi$ exist. Really, the following vector expression

$$
|u\rangle:=\exp \left\{\left(u, a^{+}\right)\right\}|\Omega\rangle,
$$

where $(.,$.$) is the standard scalar product in the Hilbert space M$, satisfies the defining condition (2.50), and moreover, the norm

$$
\|u\|_{\Phi}:=\langle u \mid u\rangle^{1 / 2}=\exp \left(\frac{1}{2}\|u\|^{2}\right)<\infty,
$$

since $u \in M$ and its norm $\|u\|:=(u, u)^{1 / 2}$ is bounded. 


\section{The Fock space Embedding method, nOnlinear DynAmical Systems And their COMPLETE} LINEARIZATION

Consider any function $u \in M:=L_{2}\left(\mathbb{R}^{m} ; \mathbb{R}^{s}\right)$ and observe that the Fock space embedding mapping

$$
\xi: M \ni u \longrightarrow|u\rangle \in \Phi
$$

defined by means of the coherent vector expression (2.51) realizes a smooth isomorphism between Hilbert spaces $M$ and $\Phi$. The inverse mapping $\xi^{-1}: \Phi \longrightarrow M$ is given by the following exact expression:

$$
u(x)=\langle\Omega|a(x)| u\rangle
$$

holding for almost all $x \in \mathbb{R}^{m}$. Owing to condition (2.52), one finds from (3.2) that, the corresponding function $u \in M$.

In the Hilbert space $M$, let now define a nonlinear dynamical system (which can, in general, be nonautonomous) in partial derivatives

$$
d u / d t=K[u]
$$

where $t \in \mathbb{R}_{+}$is the corresponding evolution parameter, $[u]:=\left(t, x ; u, u_{x}, u_{x x}, \ldots, u_{r x}\right), r \in \mathbb{Z}_{+}$, and a mapping $K: M \longrightarrow T(M)$ is Frechet smooth. Assume also that the Cauchy problem

$$
\left.u\right|_{t=+0}=u_{0}
$$

is solvable for any $u_{0} \in M$ in an interval $[0, T) \subset \mathbb{R}_{+}^{1}$ for some $T>0$. Thereby, the smooth evolution mapping is defined

$$
T_{t}: M \ni u_{0} \longrightarrow u\left(t \mid u_{0}\right) \in M
$$

for all $t \in[0, T)$.

It is now natural to consider the following commuting diagram

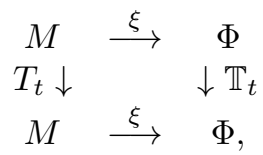

where the mapping $\mathbb{T}_{t}: \Phi \longrightarrow \Phi, t \in[0, T)$, is defined from the conjugation relationship

$$
\xi \circ T_{t}=\mathbb{T}_{t} \circ \xi
$$

Now take coherent vector $\left|u_{0}\right\rangle \in \Phi$, corresponding to $u_{0} \in M$, and construct the vector

$$
|u\rangle:=\mathbb{T}_{t} \cdot\left|u_{0}\right\rangle
$$

for all $t \in[0, T)$. Since vector $(3.8)$ is, by construction, coherent, that is

$$
a_{j}(x)|u\rangle:=u_{j}\left(x, t \mid u_{0}\right)|u\rangle
$$

for each $j=\overline{1, s}, t \in[0, T)$ and almost all $x \in \mathbb{R}^{m}$, owing to the smoothness of the mapping $\xi: M \longrightarrow \Phi$ with respect to the corresponding norms in the Hilbert spaces $M$ and $\Phi$, we derive that coherent vector (3.8) is differentiable with respect to the evolution parameter $t \in[0, T)$. Thus, one can easily find [26, 25] that

$$
\frac{d}{d t}|u\rangle=\hat{K}\left[a^{+}, a\right]|u\rangle
$$


where

$$
\left.|u\rangle\right|_{t=+0}=\left|u_{0}\right\rangle
$$

and a mapping $\hat{K}\left[a^{+}, a\right]: \Phi \longrightarrow \Phi$ is defined by the exact analytical expression

$$
\hat{K}\left[a^{+}, a\right]:=\left(a^{+}, K[a]\right) .
$$

As a result of the consideration above we obtain the following theorem.

Theorem 3.1. Any smooth nonlinear dynamical system (3.3) in Hilbert space $M:=L_{2}\left(\mathbb{R}^{m} ; \mathbb{R}^{s}\right)$ is representable by means of the Fock space embedding isomorphism $\xi: M \longrightarrow \Phi$ in the completely linear form (3.10).

We now make some comments concerning the solution to the linear equation (3.10) under the Cauchy condition (3.11). Since any vector $|u\rangle \in \Phi$ allows the series representation

$$
\begin{aligned}
|u\rangle= & \bigoplus_{n:=\sum_{j=1}^{s} n_{j} \in \mathbb{Z}_{+}} \frac{1}{\left(n_{1} ! n_{2} ! \ldots n_{s} !\right)^{1 / 2}} \int_{\left(\mathbb{R}^{m}\right)^{n}} f_{n_{1} n_{2} \ldots n_{s}}^{(n)}\left(x_{1}^{(1)}, x_{2}^{(1)}, \ldots, x_{n_{1}}^{(1)} ;\right. \\
& \left.x_{1}^{(2)}, x_{2}^{(2)}, \ldots, x_{n_{2}}^{(2)} ; \ldots ; x_{1}^{(s)}, x_{2}^{(s)}, \ldots, x_{n_{s}}^{(s)}\right) \prod_{j=1}^{s}\left(\prod_{k=1}^{n_{j}} d x_{k}^{(j)} a_{j}^{+}\left(x_{k}^{(j)}\right)\right)|\Omega\rangle,
\end{aligned}
$$

where for any $n=\sum_{j=1}^{s} n_{j} \in \mathbb{Z}_{+}$functions

$$
f_{n_{1} n_{2} \ldots n_{s}}^{(n)} \in \bigotimes_{j=1}^{s} L_{2, s}\left(\left(\mathbb{R}^{m}\right)^{n_{j}} ; \mathbb{C}\right) \simeq L_{2, s}\left(\mathbb{R}^{m n_{1}} \times \mathbb{R}^{m n_{2}} \times \ldots \mathbb{R}^{m n_{s}} ; \mathbb{C}\right)
$$

and the norm

$$
\|u\|_{\Phi}^{2}=\sum_{n=\sum_{j=1}^{s} n_{j}}\left\|f_{n_{1} n_{2} \ldots n_{s}}^{(n)}\right\|_{2}^{2}=\exp \left(\|u\|^{2}\right) .
$$

By substituting (3.13) into equation (3.10), reduces (3.10) to an infinite recurrent set of linear evolution equations in partial derivatives on coefficient functions (3.14). The latter can often be solved [25] step by step analytically in exact form, thereby, making it possible to obtain, owing to representation (3.2), the exact solution $u \in M$ to the Cauchy problem (3.4) for our nonlinear dynamical system in partial derivatives (3.3).

Remark 3.2. Concerning some applications of nonlinear dynamical systems like (3.1) in mathematical physics problems, it is very important to construct their so called conservation laws or smooth invariant functionals $\gamma: M \longrightarrow \mathbb{R}$ on $M$. Making use of the quantum mathematics technique described above one can suggest an effective algorithm for constructing these conservation laws in exact form.

Indeed, consider a vector $|\gamma\rangle \in \Phi$, satisfying the linear equation:

$$
\frac{\partial}{\partial t}|\gamma\rangle+\hat{K}^{*}\left[a^{+}, a\right]|\gamma\rangle=0
$$

Then, the following proposition [25] holds.

Proposition 3.3. The functional

$$
\gamma:=\langle u \mid \gamma\rangle
$$

is a conservation law for dynamical system (3.1), that is

$$
d \gamma /\left.d t\right|_{K}=0
$$


along any orbit of the evolution mapping (3.5).

\section{Conclusion}

Within the scope of this work we have described the main mathematical preliminaries and properties of the quantum mathematics techniques suitable for analytical studying of the important linearization problem for a wide class of nonlinear dynamical systems in partial derivatives in Hilbert spaces. This problem was analyzed in much detail using the Gelfand-Vilenkin representation theory [14] of infinite dimensional groups and the Goldin-Menikoff-Sharp theory [16, 15, 17] of generating Bogolubov type functionals, classifying these representations. The related problem of constructing Fock type space representations and retrieving their creation-annihilation generating structure still needs a deeper investigation within the approach devised. Here we mention only that some aspects of this problem within the so-called Poissonian White noise analysis were studied in a series of works [2, 1, 24, 27, based on some generalizations of the Delsarte type characters technique. It is also necessary to mention the related results obtained in [23, 25, 26], devoted to the application of the Fock space embedding method to finding conservation laws and the so called recursion operators for the well known Korteweg-de Vries type nonlinear dynamical systems. We plan to devote our next investigations to concerning some important applications of the methods devised in the work to concrete dynamical systems.

\section{ACKNOWLEDGMENTS}

Two of the authors (N.B. and A.P.) are cordially thankful to the Abdus Salam International Centre for Theoretical Physics in Trieste, Italy, for the hospitality during their research 2007-scholarships. A.P. is also thankful to Profs. Y. Kondratyev and Y. Kozicki for interesting discussions of the results during the Third International Conference on Infinite Dimensional Systems, held June 23-28, 2007 in Kazimierz Dolny, Poland, and to Profs. O. Celebi, K. Zheltukhin, G. Barsegian, O. Zhdanov and V. Golubeva during the ISAAC-2007 International Conference, held 13-18 August 2007 in Ankara, Turkey. 


\section{REFERENCES}

[1] Albeverio S., Kondratiev Y.G. and Streit L. How to generalize white noice analysis to non-gaussian measures. Preprint Bi Bo S, Bielefeld, 1992.

[2] Berezansky Y.M. A generalization of white noice analysis by means of theory of hypergroups. Reports on Math. Phys., 38, N.3 (1996), pp. 289-300.

[3] Berezansky Y.M. and Kondratiev Y.G. Spectral methods in infinite dimensional analysis, v.1 and 2, Kluwer, 1995.

[4] Bogolubov N.N. and Bogolubov N.N. (jr.) Introduction into quantum statistical mechanics. World Scientific, NJ, 1986, $384 \mathrm{P}$.

[5] Bogoliubov N.N. (Jr.), Prykarpatsky A.K. Quantum method of generating Bogolubov functionals in statistical physics: current Lie algebras, their representations and functional equations. Physics of Elementary Particles and Atomique Nucleus, v.17, N.4 (1986), pp. 791-827 (in Russian).

[6] Berezin F.A. The second quantization method. Nauka Puplisher, Moscow, 1986 (in Russian).

[7] Bogoliubov N.N. Collected works, v.2, Naukova Dumka, Kiev, 1960 (in Russian).

[8] Dirac P.A.M. The principles of quantum mechanics. Oxford University Press, 1932.

[9] Dixmier J. C*-algebras. Amsterdam, North-Holland, 1982.

[10] Faddeev L.D., Sklyanin E.K. Quantum mechanical approach to completely integrable field theories. Proceed. of the USSR Academy of Sciences (DAN), 243 (1978), pp. 1430-1433 (in Russian).

[11] Feynman R. Quantum mechanical computers. Found. Physics, 16 (1986), pp. 507-531.

[12] Feynman R. Simulating physics with computers. Intern. Journal of Theor. Physics, 21 (1982), pp. 467-488.

[13] Fock V.A. Konfigurationsraum und zweite Quantelung. Zeischrift Phys., Bd. 75 (1932), pp. 622-647.

[14] Gelfand I. and Vilenkin N. Generalized functions, 4, Academic Press, New York, 1964.

[15] Goldin G.A. Nonrelativistic current algebras as unitary representations of groups. Journal of Mathem. Physics, 12(3), 1971, pp. 462-487.

[16] Goldin G.A., Grodnik J., Powers R.T. and Sharp D. Nonrelativistic current algebra in the N/V limit. J. Math. Phys., 15, (1974), pp. 88-100.

[17] Goldin G.A., Menikoff R. and Sharp F.H. Diffeomorphism groups, gauge groups, and quantum theory. Phys. Rev. Lett. 51 (1983), pp. 2246-2249.

[18] Grover L.K. Quantum mechanics helps in searching for a needle in a haystack. Phys. Rev. Lett., 79 (1997), pp. 325-328.

[19] Jaffe A. and Quinn F. Theoretical mathematics: toward a cultural synthesis of mathematics and theoretical physics. Bull. Amer. Math. Soc., 29 (1993), pp. 1-13 Zeischrift Phys., Bd. 75 (1932), pp. 622-647.

[20] Deutsch D. Quantum theory, the Church-Turing principle and the universal quantum computer. Proc. Roy. Soc. (London), A400 (1985), pp. 97-117.

[21] Drinfeld V.G. Quantum groups. Proceed. of the Int. Congress of Mathematicians, 1986, pp. 798-820.

[22] Donaldson S.K. An application of gauge theory to four dimansional topology. J. Diff. Geom., 17 (1982), pp. 279-315.

[23] Kowalski K. and Steeb W.-H. Symmetries and first integrals for nonlinear dynamical systems: Hilbert space approach. I and II. Progress of Theoretical Physics, 85, N.4 (1991), pp. 713-722 and 85, N4 (1991), pp. 975-983.

[24] Kondratiev Y.G., Streit L., Westerkamp W. and Yan J.-A. Generalized functions in infinite dimensional analysis. II AS preprint, 1995.

[25] Kowalski K. Methods of Hilbert spaces in the theory of nonlinear dynamical systems. World Scientific, 1994.

[26] Kowalski K. and Steeb W.-H. Non linear dynamical systems and Carleman linearization. World Scientific, 1991.

[27] Lytvynov E.W., Rebenko A.L. and Shchepaniuk G.V. Wick calculus on spaces of generalized functions compound Poisson white noise. Reports on Math. Phys., 39, N.2 (1997), pp. 219-247.

[28] Manin Yu.I. Computable and uncomputable. Moscow, Sov. Radio, 1980 (in Russian).

[29] Manin Yu.I. Classical computation, quantum computation and P. Shor's factoraizing algorithm. Proceed. of the Bourbaki Seminar, 1999.

[30] Neumann J. von. Mathematische Grundlagen der Quanten Mechanik. J. springer, Berlin, 1932.

[31] Prykarpatsky A.K., Taneri U. and Bogolubov N.N. (jr.) Quantum field theory and application to quantum nonlinear optics. World Scientific, NY, 2002.

[32] Shor P.W. Polynomial time algorithms for prime factorization and discrete logarithms on a quantum computer. SIAM Journ. Comput., 26 N.5 (1997), pp. 1484-1509.

[33] Weyl H. The Theory of Groups and Quantum Mechanics. Dover, New York, 1931.

[34] Witten E. Nonabelian bozonization in two dimensions. Commun. Mathem. Physics, 92 (1984), pp. 455-472. 